\title{
CARACTERÍSTICAS CLÍNICAS, FÍSICO-FUNCIONAIS E LABORATORIAIS DE IDOSOS COM ALTO E BAIXO RISCO DE QUEDAS
}

Clinical, physical, functional and laboratory characteristics of the elderly with high and low risk of falls

Características clínicas, físico-funcionales y de laboratorio de personas mayores com alto y bajo riesgo de caídas.

\section{Emerson Moura Ribeiro ${ }^{1}$, Andreia Travassos*2, Sarah Tiane Joodsie Costa da Silva ${ }^{3}$,} Claudio Avelino Rodrigues ${ }^{4}$, Luiz Sinésio Silva Neto ${ }^{5}$, Neila Barbosa Osório ${ }^{5}$.

${ }^{1}$ Programa de Pós-graduação Mestrado em Ensino em Ciências e Saúde, Fisioterapeuta, Universidade Federal do Tocantins, Palmas, Brasil.

${ }^{2}$ Programa de Pós-graduação em Biodiversidade e Biotecnologia da rede BIONORTE, Mestre em Ciências da Reabilitação, Fisioterapeuta, Laboratório de Ciências Básicas e da Saúde, Palmas, Brasil.

${ }^{3}$ Fisioterapeuta, Docente no Instituto de Ensino e Pesquisa Objetivo, Palmas, Brasil.

${ }^{4}$ Programa de Pós-graduação Mestrado em Ensino em Ciências e Saúde, Educador Físico, Universidade Federal do Tocantins, Palmas, Brasil.

${ }^{5}$ Universidade da Maturidade, Docente do Curso de Medicina, Universidade Federal do Tocantins, Palmas, Brasil.

*Correspondência: Laboratório de Ciências Básicas e da Saúde, Complexo Laboratorial de Nutrição, Av. NS 15, 109 Norte, Palmas, Tocantins, Brasil.CEP:77.001-090.e-mail andreia-travassos@hotmail.com.

\section{Artigo recebido em 02/03/2020 aprovado em 06/11/2020 publicado em 04/03/2021.}

\section{RESUMO}

O envelhecimento favorece algumas mudanças no corpo, o que agrava o estado de saúde e gera riscos de quedas, por isso torna-se importante o estudo das características de idosos com maior risco de quedas. Este estudo procurou comparar as características clínicas, físico-funcionais e laboratoriais de idosos com baixo e alto risco de quedas, classificados pelo teste de Timed up and Go (TUG). Trata-se de um estudo transversal com amostra de conveniência, realizado na Universidade da Maturidade da Universidade Federal do Tocantins. Participaram do estudo 40 idosos que foram submetidos à avaliação da composição corporal, flexibilidade, força de preensão palmar, velocidade de caminhada e exames laboratoriais. Um ponto de corte de 12,47 s no teste TUG classificou os idosos em alto risco de quedas (ARQ) e baixo risco de quedas (BRQ), sendo que, 70\% dos idosos foram classificados como BRQ e 30\% como ARQ. Os idosos com ARQ são mais velhos, possuem maior gordura corporal e uso de polifarmácia (63\%), além de baixa flexibilidade, funcionalidade e força muscular.

Palavras-chave: envelhecimento; quedas; funcionalidade.

\section{ABSTRACT}

Aging favors some changes in the body, which aggravates the state of health and generates risks of falls, so it is important to study the characteristics of the elderly with a higher risk of falls. This study aimed to compare the clinical, physical-functional and laboratory characteristics of elderly people with low and high risk of falls, classified by the Timed up and Go (TUG) test. This is a cross-sectional study with a convenience sample, carried out at the Maturity University of the Federal University of Tocantins. Forty elderly people participated in the study, who were submitted to body composition, flexibility, handgrip strength, walking speed and laboratory exams. A cut-off point of 12.47s in the TUG test classified the elderly at high risk of falls (ARQ) and low risk of falls (BRQ), with 70\% of the elderly being classified as BRQ and $30 \%$ as ARQ. The elderly with ARQ are older, have greater body fat and use of polypharmacy (63\%), in addition to low flexibility, functionality and muscle strength.

Keywords: aging; falls; functionality. 


\section{RESUMEN}

El envejecimiento favorece algunos cambios en el cuerpo, lo que agrava el estado de salud y genera riesgos de caídas, por lo que es importante estudiar las características de las personas mayores con mayor riesgo de caídas. Este estudio buscó comparar las características clínicas, físico-funcionales y de laboratorio de las personas mayores con bajo y alto riesgo de caídas, clasificadas por la prueba Timed up and Go (TUG). Este es un estudio transversal con una muestra de conveniencia, realizado en la Universidad de Madurez de la Universidad Federal de Tocantins. Cuarenta personas mayores participaron en el estudio, quienes fueron sometidos a composición corporal, flexibilidad, fuerza de agarre, velocidad de marcha y exámenes de laboratorio. Un punto de corte de 12.47s en la prueba TUG clasificó a los ancianos con alto riesgo de caídas (ARQ) y bajo riesgo de caídas (BRQ), con el $70 \%$ de los ancianos clasificados como BRQ y el $30 \%$ como $A R Q$. Los ancianos con ARQ son mayores, tienen mayor grasa corporal y uso de polifarmacia (63\%), además de poca flexibilidad, funcionalidad y fuerza muscular.

Descriptores: envejecimiento; caídas; funcionalidade.

\section{INTRODUÇÃO}

O envelhecimento da população está relacionado ao desenvolvimento socioeconômico e epidemiológico ocasionando modificação no aspecto demográfico, redução da taxa de natalidade e aumento da expectativa de vida e do número de velhos (REZENDE et al., 2012). Tais alterações implicam na dinâmica dos sistemas de saúde que devido aumento da longevidade deve se tornar mais abrangente $\mathrm{e}$ direcional (ANDRIOLO et al., 2016).

Estudos mostram que o grande desafio enfrentado pelos velhos, em consequência do aumento da expectativa de vida são as alterações decorrentes do próprio envelhecimento, sendo elas o declínio dos órgãos e sistemas vitais do corpo, que gera redução da capacidade funcional e alteração na resposta aos estímulos do ambiente. Essas demandas podem ser internas (doenças) ou externas (situação econômica ou tipo de moradia), que levam a fragilidade e ao risco de quedas (ALMEIDA et al., 2012; DUARTE et al., 2019), que é definido como "um evento não intencional que leva o indivíduo a um nível mais baixo em relação a postura inicial" (DUARTE et al., 2019).

Pessoas de todas as idades estão sujeitas a quedas. Esse evento promove agravos relevantes no idoso quando associado ao sedentarismo, a uma saúde debilitada e ao maior consumo de medicamentos. A ocorrência desse evento é multifatorial e depende de alguns dos fatores intrínsecos e extrínsecos para ocorrer (STREIT et al., 2012). Todavia, as quedas geram consequências graves como fraturas, lesões dos tecidos moles, hospitalizações além do alto risco de mortalidade (CAMPOS et al.,2013).

Só em 2011, aproximadamente $980 \mathrm{mil}$ internações foram provocadas por quedas relacionadas a fatores ambientais com um gasto equivalente a 131 bilhão de reais, financiado pelo Sistema Único de Saúde, sendo responsável por cerca de 152 milhões de óbitos no Brasil em 2013 (ABREU et al., 2018).

Nesse sentido, a identificação de variáveis clínicas, físico-funcionais e laboratoriais que possam estar associadas a quedas nos idosos pode influenciar o direcionamento das intervenções de promoção e prevenção em saúde, reduzir índices de mortes e gastos públicos, possibilitando que o aumento da longevidade não ocasione piora da qualidade de vida. Dessa forma, esse trabalho tem o objetivo de comparar as características clínicas, físico-funcionais e laboratoriais de idosos com baixo e alto risco de quedas classificados a partir do teste Timed Up and Go (TUG).

\section{MATERIAIS E MÉTODOS}

\section{Delineamento e amostra}

Esse trabalho trata-se de um estudo transversal

com amostra de conveniência realizado na Universidade da Maturidade (UMA) da Universidade 
Federal do Tocantins (UFT) em outubro de 2018. Participaram do presente estudo 40 idosos sendo 31 mulheres e 9 homens. Como critério de inclusão foi considerado idade $\geq$ a 60 anos, assinatura do Termo de Consentimento Livre e Esclarecido e ausência de contraindicações e limitações ortopédicas e/ou cognitivas para a realização dos protocolos de pesquisa propostos.

\section{Aspectos éticos}

Os dados utilizados foram extraídos de um projeto de pesquisa longitudinal da UMA/UFT, devidamente aprovado pelo Comitê de Ética da instituição sob o Certificado de Apresentação para Apreciação Ética (CAAE) número 72784016.4.0000.5519.

\section{Avaliações e instrumentos}

Após serem incluídos no estudo, os indivíduos foram avaliados quanto a dados clínicos (idade, sexo, multimorbidade e polifarmácia), laboratoriais, avaliação antropométrica e de composição corporal, nível de força, flexibilidade e funcionalidade por meio do teste de TUG e de velocidade da marcha (VM).

Para a análise antropométrica foi mensurado o peso corporal em quilogramas $(\mathrm{kg})$ na posição vertical em balança mecânica e a estatura em metros por meio de uma régua com precisão de $0,5 \mathrm{~cm}$. $\mathrm{O}$ índice de massa corporal (IMC) foi calculado pelo peso em $\mathrm{kg}$ dividido pela altura ao quadrado.

Os dados laboratoriais foram adquiridos por coleta de sangue após jejum de 8 horas. Foram realizadas análises em relação ao perfil lipídico, marcadores de função hepática, renal e muscular por meio de padrões comerciais.

A fim de mensurar composição corporal, utilizou-se o equipamento de Bioimpedância InBody
$370 ®$, para verificar massa músculo esquelética, massa livre de gordura e massa gorda cujos valores absolutos foram normalizados pela altura do indivíduo. Para controlar os fatores que poderiam afetar os resultados desse exame, os idosos não deveriam consumir álcool nas 48 horas precedentes do teste; não consumir produtos com propriedades diuréticas como cafeína ou chocolate nas 24 horas precedentes; não praticar exercícios físicos intensos 12 horas antes; estar em jejum de pelo menos 4 horas; e esvaziar completamente a bexiga 30 minutos antes do procedimento.

A avaliação da força muscular foi mensurada pelo dinamômetro Jamar®, por meio da força de preensão palmar (FPP). O dinamômetro foi calibrado e regulado para cada medida e, em seguida, os indivíduos foram assentados numa cadeira sem apoio para os braços, pés apoiados no chão com quadril e joelho posicionados a $90^{\circ}$, antebraço neutro e punho entre 0 e $30^{\circ}$ de extensão, com adução de 0 a $15^{\circ}$, com a mão contralateral apoiada sobre a coxa ipsilateral. Os indivíduos não deveriam usar acessórios no braço avaliado. Cada idoso foi orientado a realizar uma contração isométrica de 3 segundos sob comando verbal constante, de forma não alternada. A análise foi feita nas 2 mãos por 3 vezes de cada lado, com intervalos de descanso de 30 segundos entre cada contração do mesmo lado e de 2 minutos entre os membros. Ao final, escolheu-se o maior valor mensurado nos dois braços (VERSIANI et al., 2010).

A funcionalidade foi avaliada pelo teste de TUG, que consiste na tarefa de levantar de uma cadeira, caminhar por três metros e retornar a mesma. Os indivíduos só foram submetidos a avaliação depois de familiarizar-se com o percurso, que não deveria ser executado com ajuda ou apoio. Para a classificação dos idosos em alto ou baixo risco de quedas, foi utilizado o valor preditor $\geq 12,47 \mathrm{~s}$ para alto risco de quedas $\mathrm{e} \leq$ 
12,47s pra baixo risco de quedas (ALEXANDRE et al., 2012).

Para a avaliação da VM, o idoso deveria percorrer uma distância de 4 metros procurando andar em sua velocidade habitual, com o tempo controlado por um cronômetro digital Casio ${ }^{\circledR} \mathrm{HS}-3 \mathrm{~V}-1$. O valor da velocidade foi mensurado pela relação entre a distância percorrida e o tempo em segundos.

Para mensurar a flexibilidade aplicou-se o teste de sentar-se e alcançar. O indivíduo teve que se sentar com os joelhos estendidos e a superfície plantar dos pés apoiadas contra uma caixa com $30,3 \mathrm{~cm} \times 30,5 \mathrm{~cm} \times$ $30,5 \mathrm{~cm}$. Com as mãos sobrepostas, os indivíduos foram orientados a flexionar o tronco a frente procurando mover com as mãos a escala até o ponto máximo possível. A mensuração foi realizada 3 vezes considerando a de melhor resultado (RIBEIRO et al., 2010).

\section{Análise estatística}

$\mathrm{Na}$ avaliação da normalidade dos dados, foi utilizado o teste de Shapiro-wilk. Os dados classificados como normais foram descritos como média \pm desvio padrão e os não normais como mediana [intervalo interquartílico 25-75\%]. As características dos indivíduos com alto e baixo risco de quedas foram comparadas por meio do teste T não pareado ou MannWhitney, enquanto as variáveis categóricas foram comparadas pelo teste de Qui-quadrado. A tabulação dos dados foi realizada por meio do software Microsoft Excel e a análise estatística por meio do software SPSS versão 22.0. A significância estatística adotada foi de $\mathrm{P}<0,05$.

\section{RESULTADOS E DISCUSSÃO}

As quedas nos idosos são eventos multifatoriais, incapacitantes e associados a maior mortalidade. Nesse sentido, o conhecimento de um fenótipo relacionado às quedas poderá influenciar nas estratégias de prevenção e tratamento dessa população (GASPAROTTO et al., 2014; MORAES et al., 2018). Dessa maneira, de acordo com a comparação das características clínicas, físicas, funcionais e laboratoriais de idosos com alto e baixo risco de quedas foi observado no presente estudo, que indivíduos com maior risco de quedas são mais velhos, apresentam redução da funcionalidade, maior gordura corporal, redução da força muscular e maior índice de polifarmácia.

Nesse aspecto, ALMEIDA et al. (2012) apontam que quanto maior a idade, mais propenso a quedas o idoso estará. Logo, esse argumento corrobora com os resultados do presente estudo (Tab.1) no qual os indivíduos com ARQ possuíam maior idade.

Tabela 1. Características da amostra.

\begin{tabular}{lccc}
\hline \multicolumn{1}{c}{ Variáveis } & \multicolumn{2}{c}{ Amostra $(\mathbf{n = 4 0})$} & P \\
\hline Risco de quedas & $\mathrm{ARQ}=12$ & $\mathrm{BRQ}=28$ & - \\
& $(30 \%)$ & $(70 \%)$ & \\
Idade & 68 & 64,5 & $0,029 *$ \\
& {$[67-74,75]$} & {$[62-70]$} & \\
Sexo & $\mathrm{M}=10$ & $\mathrm{M}=21$ & 0,563 \\
& $(83,3 \%)$ & $(75,0 \%)$ & \\
Multimorbidade & $\mathrm{Sim}=7$ & $\mathrm{Sim}=8$ & 0,314 \\
& $(45,5 \%)$ & $(28,6 \%)$ & \\
Polifarmácia & $\mathrm{Sim}=5$ & $\mathrm{Sim}=3$ & $0,001^{* *}$ \\
& $(63,6 \%)$ & $(10,7 \%)$ & \\
\hline
\end{tabular}

ARQ: Alto risco de quedas; BRQ: Baixo risco de quedas; M: mulheres; H: homens; * Teste de Mann-Whitney; **Qui-quadrado

Tais evidências se associam com a redução da funcionalidade por consequência das alterações típicas do envelhecimento, como a redução do controle postural, adaptação com o ambiente, transtornos cognitivos além da incapacidade de recuperar ou manter o equilíbrio que ocasionalmente predispõe a quedas (VALDUGA et al., 2016).

Além disso, um alto risco de quedas também está associado com maior consumo de medicamentos, 
visto que a ingestão de 4 ou mais fármacos (hipoglicêmicos, anti-hipertensivo e outros) podem gerar déficits motores, fadiga, fraqueza, vertigem e hipotensão postural levando ao risco de quedas (SOUZA et al., 2017). Compartilhando dessa mesma observação, REZENDE et al. (2012) ao estudar quedas em idosos brasileiros e sua relação com o uso de medicamentos, acrescentou também que estes possam gerar sedação, tremores e relaxamento muscular.

Ademais, em relação a incidência de multimorbidade não houve diferenças entre os grupos. Tal resultado pode ter sofrido interferência de uma possível prática de exercício físico (EF) pelos indivíduos com BRQ. Segundo YABUUTI et al. (2019) a prática de EF pode propiciar maior independência e menor número de comorbidades, contudo o nível de atividade física não foi avaliado no estudo.

A literatura aponta que mulheres caem mais ao comparar-se com homens. A relação de fatores extrínsecos e intrínsecos com uma menor quantidade de massa magra, força muscular e alterações hormonais típicas da menopausa, deixaria a mulher mais frágil e levaria a quedas (REZENDE et al., 2012; FERREIRA \& YOSHITOME, 2010). Porém, na presente pesquisa, não houve diferença na proporção de homens e mulheres com ARQ e BRQ. A comparação do risco de quedas com o sexo não foi avaliada.

Além disso, ao comparar a incidência/risco de quedas de idosos apontadas literaturas, fica evidente que, no geral, os indivíduos dessa pesquisa foram menos propensos a quedas pois apenas 30\% da amostra tinha alto risco de quedas. No estudo de ALMEIDA et al. (2019), as incidências de quedas foram $60 \%$ para mulheres e $40 \%$ em homens, assim como SILVA et al. (2013) verificaram um risco de quedas de $47 \%$ em sua amostra.
Ao comparar variáveis laboratoriais, não houve diferença para marcadores de perfil lipídico (PL) (triglicerídeos, colesterol total, lipoproteína de alta densidade, lipoproteína de baixa densidade, colesterol não HDL e lipoproteínas de muito baixa densidade) entre os grupos. No entanto, estudos apontam que níveis alterados no PL se associam a fatores de risco para doenças e esses poderiam levar a incapacidades do tipo respiratórias, músculo esqueléticas, doenças cardiovasculares e consequentemente a quedas (YABUUTI et al., 2019).

Também foi encontrado no presente estudo que idosos com ARQ (Tab.2), possuem menores níveis de creatinafosfoquinase $(\mathrm{CPK})$, conhecida como melhor marcador de função muscular. Seu aumento ocorrerá quando houver lesão nas células desse tecido (CRUZ, 2011). Com isso, níveis reduzidos de CPK presentes no ARQ, poderia expressar a ideia de que idosos com BRQ usam a musculatura de forma mais funcional a ponto de estimular alterações bioquímicas nas fibras musculares. Outrossim, idosos com ARQ apresentaram menor nível de creatina fosfatase $(\mathrm{PCr})$. Com isso, é possível sugerir que níveis reduzidos de $\mathrm{PCr}$ promovam déficits na contratilidade muscular, pois a PCr é produzida nos períodos de repouso e de atividade muscular após o processo de fosforilação (FERREIRA, 2014).

Pode-se observar diferenças significativas para marcadores de função hepática e renal (TGP transaminase glutâmico-pirúvica, TGO - transaminase glutâmico-oxalacética e AU - ácido úrico), dessa forma, o maior nível de AU com menores níveis de TGP e TGO no grupo com ARQ, indicaria uma menor função hepática e renal pela baixa filttração glomerular em função do declínio da idade (PINTO, 2010; TONELLI \& RIELLA, 2014). 
Tabela 2. Comparação de variáveis laboratoriais entre grupos ARQ e BRQ.

\begin{tabular}{|c|c|c|c|}
\hline Variável & $\begin{array}{c}\text { ARQ } \\
(n=12)\end{array}$ & $\begin{array}{c}\text { BRQ } \\
(n=28)\end{array}$ & $\mathbf{P}$ \\
\hline $\begin{array}{l}\text { Glicemia } \\
(\mathrm{mg} / \mathrm{dl})\end{array}$ & $\begin{array}{c}106 \\
{[80,5-132,5]}\end{array}$ & $\begin{array}{c}84 \\
{[76,7-89]}\end{array}$ & 0,029 \\
\hline $\begin{array}{l}\text { Creatina } \\
\text { fosfatase }(\mathrm{u} / \mathrm{l})\end{array}$ & $2[2-2]$ & $3[2-3]$ & $0,017 *$ \\
\hline $\begin{array}{l}\text { Triglicerídeos } \\
(\mathrm{mg} / \mathrm{dl})\end{array}$ & $\begin{array}{c}161 \\
{[93,2-192]}\end{array}$ & $\begin{array}{c}98 \\
{[71-124]}\end{array}$ & 0,06 \\
\hline $\begin{array}{l}\text { Colesterol total } \\
(\mathrm{mg} / \mathrm{dl})\end{array}$ & $19358 \pm 63,9$ & $196,3 \pm 45,1$ & 0,631 \\
\hline HDL (mg/dl) & $42,4 \pm 10,4$ & $47,9 \pm 14,3$ & 0,243 \\
\hline LDL (mg/dl) & $121,4 \pm 52,1$ & $125,9 \pm 4,5$ & 0,553 \\
\hline TGP (u/l) & $\begin{array}{c}12 \\
{[10-17]}\end{array}$ & $\begin{array}{c}19 \\
{[14,5-33,2]}\end{array}$ & $0,012 *$ \\
\hline TGO (u/l) & 16 [14-19] & 22 [17-30] & $0,002^{*}$ \\
\hline CPK (u/l) & $\begin{array}{c}85 \\
{[64-96]}\end{array}$ & $\begin{array}{c}102 \\
{\left[\begin{array}{ll}77 & 145\end{array}\right]}\end{array}$ & $0,027^{*}$ \\
\hline $\begin{array}{l}\text { Colesterol não } \\
\text { HDL (mg/dl) }\end{array}$ & $146,3 \pm 60,2$ & $141 \pm 58,8$ & 0,999 \\
\hline VLDL (mg/dl) & $\begin{array}{c}32,2 \\
{[18,6-38,4]}\end{array}$ & $\begin{array}{c}19,6 \\
{[14,2-24,8]}\end{array}$ & 0,061 \\
\hline $\begin{array}{l}\text { Ácido Úrico } \\
(\mathrm{mg} / \mathrm{dl})\end{array}$ & $5,12 \pm 1,47$ & $4,18 \pm 1,13$ & $0,021 * *$ \\
\hline $\begin{array}{l}\text { Creatinina } \\
(\mathrm{mg} / \mathrm{dl})\end{array}$ & $\begin{array}{c}0,9 \\
{[0,72-1,07]}\end{array}$ & $\begin{array}{c}0,8 \\
{[0,7-1]}\end{array}$ & 0,230 \\
\hline
\end{tabular}

HDL: lipoproteína de alta densidade; LDL: lipoproteína de baixa densidade; TGP: transaminase glutâmicopirúvica; TGO: transaminase glutâmico-oxalacética; CPK: Creatinafosfoquinase; VLDL: lipoproteínas de muito baixa densidade; *Teste t não pareado; **Teste de Mann-Whitney.

As mudanças na composição corporal são fenômenos metabólicos naturais do envelhecimento e, indivíduos com idade acima de 45 anos possuem apenas $30 \%$ de massa muscular corporal (FALSARELA et al., 2014). Nesse aspecto, observa-se que idosos com ARQ são portadores de maior gordura corporal se comparados com o grupo BRQ (Tab.3). Ao considerar o IMC do grupo ARQ $(28,27 \mathrm{~kg} / \mathrm{m} 2)$ nota-se um considerável sobrepeso quando comparado ao valor de referência classificado pelo Sistema de Vigilância Alimentar e Nutricional (IMC> a 27kg/m2) (FAGUNDES et al., 2011).
Tabela 3. Comparação das variáveis de composição corporal entre grupos ARQ e BRQ.

\begin{tabular}{|c|c|c|c|}
\hline Variável & $\operatorname{ARQ}(n=12)$ & BRQ $(n=28)$ & $\mathbf{P}$ \\
\hline $\begin{array}{l}\text { IMC } \\
(\mathrm{kg} / \mathrm{m} 2)\end{array}$ & $28,27 \pm 7,78$ & $25,26 \pm 4,21$ & 0,01 \\
\hline RCQ (cm) & $0,92 \pm 0,05$ & $0,91 \pm 0,09$ & $0,007 * *$ \\
\hline $\begin{array}{l}\text { \% gordura } \\
(\mathrm{Kg})\end{array}$ & $\begin{array}{c}42,4 \\
{[36,1-49,4]}\end{array}$ & $\begin{array}{c}34,3 \\
{[25,2-42]}\end{array}$ & $0,017 *$ \\
\hline $\begin{array}{l}\text { IMLGA } \\
(\mathrm{kg})\end{array}$ & $\begin{array}{c}6,61 \\
{[5,65-6,84]}\end{array}$ & $\begin{array}{c}6,62 \\
{[5,76-7,13]}\end{array}$ & 0,738 \\
\hline $\begin{array}{l}\text { IMLG } \\
(\mathrm{kg})\end{array}$ & $\begin{array}{c}16,8 \\
{[14,6-18,4]}\end{array}$ & $\begin{array}{c}16,7 \\
{[14,8-17,4]}\end{array}$ & 0,716 \\
\hline IME & $\begin{array}{c}9,08 \\
{[7,76-9,95]}\end{array}$ & $\begin{array}{c}8,92 \\
{[7,81-9,44]}\end{array}$ & 0,805 \\
\hline $\begin{array}{l}\text { IMGA } \\
(\mathrm{kg})\end{array}$ & $5,30 \pm 3,09$ & $3,77 \pm 1,82$ & $0,008^{*}$ \\
\hline $\begin{array}{l}\text { IMGT } \\
(\mathrm{kg})\end{array}$ & $11,81 \pm 5,82$ & $8,45 \pm 4,05$ & $0,005^{*}$ \\
\hline $\begin{array}{l}\text { TMB } \\
\text { (kcal) }\end{array}$ & $1271,8 \pm 106,5$ & $1252 \pm 130,8$ & 0,765 \\
\hline \multicolumn{4}{|c|}{$\begin{array}{l}\text { IMC: Índice de massa corporal; RCQ: relação cintura- } \\
\text { quadril; IMLGA: índice de massa livre de gordura } \\
\text { apendicular; IMLG: índice de massa livre de gordura; } \\
\text { IME: índice de massa esquelética; IMGA: índice de } \\
\text { massa de gordura apendicular; IMGT: índice de massa de } \\
\text { gordura total; TMB: taxa metabólica basal; *Teste t não } \\
\text { pareado; **Teste de Mann-Whitney. }\end{array}$} \\
\hline
\end{tabular}

O IMC segundo COSTA et al. (2013) pode indicar baixo estado nutricional e maior risco de quedas, em consequência do peso desproporcional a ser suportado pelo corpo podendo afetar o equilíbrio que já está comprometido pelo avanço da idade.

No que diz respeito as medidas de RCQ que também apresentaram diferenças entre os grupos, destaca-se que já foi observado na literatura relação entre gordura abdominal e risco de quedas. $\mathrm{O}$ estudo de NERI et al. (2016) considerou o valor $>88 \mathrm{~cm}$ de circunferência abdominal como preditor para obesidade, e destacou que quanto mais gordura o indivíduo tiver, maior risco de quedas apresentará. Além do mais, PAULA et al. (2016) descrevem que com o avanço da idade ocorre redução da força, massa e função muscular acompanhada do aumento de gordura corporal, definido como sarcopenia; isso por 
sua vez leva a incapacidades funcionais (SANTANA $e t$ $a l$. , 2019). No entanto, mesmo não investigando diretamente a sarcopenia nesse estudo, o grupo com ARQ apresentou menor força muscular, que atualmente é considerado como o principal marcador de sarcopenia, acometendo a funcionalidade $\mathrm{e}$ predispondo a quedas (SANTANA et al., 2019; PAULA et al., 2016).

$\mathrm{Na}$ análise da funcionalidade (Tab.4), idosos com ARQ necessitaram de maior tempo para realizar o teste de TUG e apresentaram menor velocidade da marcha (VM), flexibilidade e força muscular se comparados com indivíduos de BRQ. Essas características são perdidas durante o envelhecimento e prejudicam a execução de tarefas cotidianas levando a quedas (LIMA, 2011).

Ao analisar o tempo para realização do TUG entre os grupos, percebe-se semelhança entre o tempo

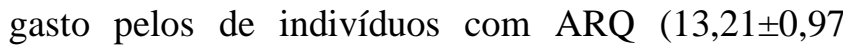
segundos) e o encontrado no estudo multidimensional de ALMEIDA et al. (2012) que identificou correlação entre idade e risco de quedas, logo, quanto maior a idade, mais elevado será o tempo de execução do TUG.

Segundo DUARTE et al. (2019) a ocorrência de quedas em idosos está interligado a redução da FPP como também a redução da VM. Essa observação corrobora com a menor FPP encontrada no grupo com ARQ (Tab.4). Com isso, idosos com maior risco de quedas tendem a possuir menor força muscular (VERSIANI et al., 2010). Além disso, o excesso de peso é citado como um bom preditor de limitação da mobilidade e da função física, pois aumenta a demanda energética durante a deambulação em atividades funcionais, modificando a biomecânica da marcha reduzindo o comprimento da passada e por fim, a velocidade da caminhada (VERSIANI et al., 2010).
Tabela 4. Comparação de características físico-funcionais entre grupos ARQ e BRQ.

\begin{tabular}{lccc}
\hline \multicolumn{1}{c}{ Variável } & ARQ $(\mathbf{n}=\mathbf{1 2})$ & BRQ $(\mathbf{n = 2 8})$ & P \\
\hline TUG (s) & $13,21 \pm 0,97$ & $9,66 \pm 1,27$ & $0,00^{* *}$ \\
VM (m/s) & $0,7[0,7-0,8]$ & $0,8[0,8-1]$ & $0,009^{*}$ \\
Flexibilidade & $21,50 \pm 8,22$ & $27,62 \pm 8,55$ & $0,017^{* *}$ \\
$(\mathrm{~cm})$ & & & \\
FPP $(\mathrm{kgf})$ & $27,08 \pm 5,53$ & $31,46 \pm 5,71$ & $0,046 * *$ \\
\hline VM: velocidade da marcha; & FPP: força de & preensão \\
palmar; *Teste t não pareado; **Teste de Mann-Whitney.
\end{tabular}

Outrossim, idosos com ARQ, possuem menor flexibilidade quando comparados com o grupo BRQ. Assim, a flexibilidade durante a velhice, sofre perda gradativa pela redução da amplitude articular a depender da pele, tendões, ligamentos e músculos podendo implicar na autonomia funcional do idoso e no risco de quedas (LIMA, 2011). Logo, quanto menos flexível for o indivíduo, maiores serão as dificuldades para lidar com tarefas cotidianas, pois o encurtamento da musculatura representa um fator limitante na 36 destreza de movimentos (LIMA, 2011).

Uma possível limitação do estudo a ser levantada é a intervenção da educação em saúde nos idosos incluídos nesse estudo. Essa amostra faz parte do programa Universidade da Maturidade da UFT, que dentre outras atividades proporciona educação em saúde para seus alunos, os idosos. Dessa forma, estimase que indivíduos orientados apresentem menores riscos de quedas por consequência do acesso a informações sobre a auto percepção de saúde, o que foi encontrado ao comparar-se a proporção de risco de quedas desse estudo com outros achados da literatura (ALMEIDA et al., 2019; SILVA et al., 2013). Contudo, a educação em saúde proporcionada pela UMA induziria a mudanças significativas nos hábitos de vida bem como na modificação de fatores que favoreçam a redução dos riscos de quedas. 


\section{CONCLUSÃO}

Por fim, conclui-se que indivíduos com ARQ possuem maior idade, gordura corporal e uso de polifarmácia, além de menor flexibilidade, funcionalidade, força muscular e função renal. Desta forma, a identificação de fatores relacionados com o risco de quedas, poderia interferir no direcionamento de intervenções com o intuito de manter condições favoráveis na saúde do idoso. Logo, as variáveis apresentadas podem melhorar as abordagens voltadas para área da gerontologia além de melhorar a qualidade de vida desses indivíduos.

\section{AGRADECIMENTO}

Agradecemos aos idosos e coordenadores da UMA pelo carinho e apoio durante a elaboração desse projeto.

Todos os autores declararam não haver qualquer potencial conflito de interesses referente a este artigo.

\section{REFERÊNCIAS}

REZENDE, C.P.; CARRILLO, M.R.G.G. e SEBASTIÃO, E.C.O. Queda entre idosos no Brasil e sua relação com o uso de medicamentos: revisão sistemática. Cad. Saúde Pública Rio de Janeiro. vol.28, n.12, p.2223-2235, 2012.

ANDRIOLO, B.N.G.; SANTOS, N.V; VOLSE, A.A.; FÉ, L.C.M.; AMARAL, A.R.C; CARMO, B.M.S.S.; CORTEZ, P.C.; GUTIERRES, D.S.; FERREIRA, L.B.M.A.F.; CARVALHO A.B.P.N. Avaliação do grau de funcionalidade em idosos usuários de um centro de saúde. Rev Soc Bras Clin Med. v.14, n.3, p.139-44, 2016.

DUARTE, G.P.; SANTOS, J.L.F.; LEBRÃO, M.L.; DUARTE, Y.A.O. Relação de quedas em idosos e os componentes de fragilidade. Rev Bras Epidemiol. v.21, 2019.

STREIT, I.A.; MAZO, G.Z.; VIRTUOSO, J.F.; MENEZES, E.C.; GONÇALVES, E. Aptidão física e ocorrência de quedas em idosos praticantes de exercícios físicos. Rev Bras Atividade Física Saúde. v.16, n.4, p.346-52, 2012.
CAMPOS, M.P.S.; VIANNA, G.L.; CAMPOS, A.R. The balancing functional reach and times up and go test risk of falls in the elderly. Rev Kairós Gerontol. v.16, n.4, p.125-38, 2013.

ABREU, D.R.O.M.; NOVAES, E.S.; OLIVEIRA, R.R.; MATHIAS, T.A.F.; MARCON, S.S. Internação e mortalidade por quedas em idosos no Brasil: análise de tendência. Cien Saude Colet. v.23, n.4, p.1131-41, 2018.

VERSIANI, L.C.; SILVEIRA, E.C.B.R.; PIMENTA, M.C.; ALVARENGA, S.G.; PARENTONI, A.N.; SAMORA, G.A.R.; FILHO, I.T.C. Influência da circunferência abdominal sobre o desempenho funcional de idosas. Fisioterapia e Pesquisa, São Paulo. v.17, n.4, p. 317-21, 2010.

ALEXANDRE, T.S.; MEIRA, D.M.; RICO, N.C.; MIZUTA, S.K. Acurácia do Timed Up and Go Test para rastrear risco de quedas em idosos da comunidade.

Brazilian J Phys Ther. v.16, n.5, p.381-8, 2012.

RIBEIRO, C.C.A.; ABAD, C.C.C.; BARROS, R.V.; NETO, T.L.B. Nível de flexibilidade obtida pelo teste de sentar e alcançar a partir de estudo realizado na Grande São Paulo. Rev Bras Cineantropometria e

Desempenho Hum. v.12, n.6, p.415-21, 2010.

GASPAROTTO, L.P.R.; FALSARELLA, G.R.; COIMBRA, A.M.V. As quedas no cenário da velhice: conceitos básicos e atualidades da pesquisa em saúde. Rev Bras Geriatr e Gerontol. v.17, n.1, p.201-209, 2014.

MORAES, E.M.; PEREIRA, A.M.V.B.; AZEVEDO, R.S.; MORAES, F.L. 2017. Avaliação miltidimensional do idoso. Secretaria de Estado da Saúde do Paraná. Curitiba, p.113.

VALDUGA， R.; LOPES, B.S.; FARIAS, D.L.; NASCIMENTO, D.C.; VIEIRA, D.C.L.; VALDUGA, L.V.A.; PRESTES, J.; CARVALHO, G.A. Risco de quedas e sua relação com a funcionalidade e medo de cair em idosas. R. bras. Ci. e Mov. v.24, n.1, p.153166, 2016.

SOUZA, L.H.R.; BRANDÃO, J.C.S.; FERNANDES, A.K.C.; CARDOSO, B.L.C. Quedas em idosos e fatores de risco associados. Rev. Aten. Saúde. v. 15, n. 54, p. 55-60, 2017.

YABUUTI, P.L.K.; JESUS, G.M.; BURATTI, A.; BASSANI, G.A.; CASTRO, H.; PEREIRA, J.S.; NAKAMOTO, J.M.; GUSHIKEN, E.S.; FILHO, C.B.; GONÇALVES, I.O. O exercício físico na terceira idade 
como instrumento de promoção da saúde. Rev Eletrônica Acervo Saúde. v.11, n.6, 2019.

FERREIRA, D.C.O.; YOSHITOME, A.Y. Prevalência e caraterísticas das quedas de idosos institucionalizados. Rev Bras Enferm, Brasília. v.63, n.6, p.991-7, 2010.

ALMEIDA, M.M.; PESSOA, R.M.C.; SANTOS, T.S. Causas e consequências de quedas de idosos atendidos em hospital público. R. Interd. v.12, n.1, p. 15-22, 2019.

SILVA, J.M.N.; BARBOSA, M.F.S.; CASTRO, P.O.C.N.; NORONHA, M.M. Correlação entre o risco de queda e autonomia funcional em idosos institucionalizados. Rev Bras Geriatr e Gerontol. v.16, n.2, p.337-346, 2013.

CRUZ, J. Indicadores Bioquímicos da Função Muscular. 2011. Univiversidade Federal do Rio Grande do Sul.

FERREIRA, L.C. Papel do sistema da fosfocreatina na homeostase energética das musculaturas esquelética e cardíaca. Einstein, Sao Paulo. v.12, n.1, p.126-31, 2014.

PINTO, S.B. Comparação entre dosagens de AST (aspastato aminotrabsferase) e ALT (amina aminotransferase) em presença e na ausência de piridoxal fosfato. Porto Alegre-RS. Dissertação de Graduação. Universidade Federal do Rio grande do Sul; 2010.

TONELLI, M.; RIELLA, M. Doença Renal Crônica e o envelhecimento da população. J Bras Nefrol. v.36, n.1, p.1-5, 2014.

FALSARELA, G.R.; GASPAROTO, L.P.R.; COIMBRA. I.B.; COIMBRA, A.M.V.
Envelhecimento e os fenótipos da composição corporal. Rev Kairós Gerontol. v.17, n.2, p.57-77, 2014.

FAGUNDES, A.; NILSON, E.A.; COUTINHO, J.G.; DUAR, H.A.; OLIVEIRA, K.S.; AQUINO, K.; TOTAL, N. Orientaçõe para a coleta e análise de dados antropométricos em serviços de saúde. 2011. Norma técnica do sistema de vigilância alientar e nutricional SISVAN. Ministério da Saúde. p.1-71.

COSTA, A.G.S.; COSTA, F.B.C.; SILVA, V.M.; ARAUJO, T.L. Ocorrência de quedas e índice de massa corporal em idosos. Rev. enferm. UERJ. v.21, n.4, p.508-514, 2013.

NERI, S.G.R.; JÓIA. L.; KAWANO, M.M. A obesidadae pode aumentar o risco de quedas em mulheres idosas. Revista das Ciências da Saúde do Oeste Baiano. v.1, n.1, p.60-72, 2016.

PAULA, J.A.; WAMSER, E.L.; GOMES, A.R.S.; VALDERRAMAS, S.R.; NETO, J.C.; SCHIEFERDECKER, M.E.M. Analysis of methods for detecting sarcopenia in independent communitydwelling elderly women. Rev Bras Geriatr e Gerontol. v.19, n.2, p.235-246, 2016.

SANTANA, N.M.; MENDES, R.M.L.; SILVA, N.F.D.; PINHO, C.P.S. Sarcopenia and sarcopenic obesity as prognostic predictors in hospitalized elderly patients with acute myocardial infarction. Einstein, São Paulo. v.17, n.4, p.1-9, 2019.

LIMA, M.S.D.; SIQUEIRA, J.E. Relação da força, da flexibilidade e do nível de atividade física no equilíbrio motor em idosos. Curitiba-PR. Dissertação de graduação. Universidade Tecnológica Federal do Paraná - UTFPR; 2011. 